\title{
Comparación de dos protocolos de superovulación en donantes de embriones Braford
}

\author{
Yostar, E.J.'; Mujica, I.F. ${ }^{2}$; Stahringer, R.C. ${ }^{3}$; Capellari, A. ${ }^{4}$ \\ ${ }^{1}$ Maestría Prod. Anim. Subtrop., Fac.Cs.Vet.Univ.Nac.Nord., Corrientes, Argentina. \\ ${ }^{2}$ IMTESA (Urdinarrain, Entre Ríos). ${ }^{3}$ EEA INTA Col.Benítez, Chaco. \\ ${ }^{4}$ Cát. Prod. Bovina FCV-UNNE. \\ E-mail: jonayostar@gmail.com
}

\begin{abstract}
Resumen
Yostar, E.J.; Mujíca, I.F.; Stahringer, R.C.; Capellari, A.: Comparación de dos protocolos de superovulación en donantes de embriones Braford. Rev. Vet. 32: 2, 146-150, 2021. La transferencia embrionaria es el proceso de colectar embriones (EMB) de una determinada hembra donante $(\mathrm{DN})$ y transferirlos a otras receptoras para continuar la gestación. Los tratamientos superovulatorios (TS) se caracterizan por provocar respuestas muy variables por lo cual es imposible estimar el número de EMB que se recupera por DN. En base a la problemática referida a la gran variabilidad que presentan los TS, como así también basado en un ensayo preliminar, se propone comenzar la super ovulación (SPO) el día 3 después de sincronizar la nueva onda folicular. El objetivo de este estudio fue evaluar la respuesta a dos TS en DN de la región Noreste Argentino. Se trabajó con 60 DN Braford de 5 cabañas asignadas aleatoriamente a dos TS (T1: $n=30$ y T2: $n=30)$, en cada programa de colectas se trabajó con 12 DN. En T1 la SPO comenzó el día 3 (considerando día 0 al momento de la colocación de dispositivo de progesterona y $17 \beta$ estradiol) acortando de esta manera todo el tratamiento y concluyendo con la colecta de EMB el día 14 y no el 15 como en T2. Las variables analizadas fueron: estructuras totales subdivididas en: EMB transferibles, degenerados y ovocitos sin fertilizar. El número de EMB transferibles logrado fue de $7 \pm 1,19$ y $6 \pm 0,98$; EMB degenerados de $1 \pm 0,32$ y $1 \pm 0,24$ y ovocitos sin fertilizar de $3 \pm 0,86$ y $7 \pm 0,83$ en T1 y $\mathrm{T} 2$, respectivamente. La prueba de independencia arrojó evidencia estadísticamente significativa para declarar la asociación entre los tratamientos y las estructuras recuperadas en la colecta, con una frecuencia de $62 \%$ de EMB transferibles en T1 y $44 \%$ en T2 ( $<<0,0001)$. Se concluye que si bien el uso anticipado de hormona folículo-estimulante (FSH) en los TS no permitió mejorar la respuesta superovulatoria, puede ser utilizado estratégicamente sin afectar la producción de embriones.
\end{abstract}

Palabras clave: bovinos, transferencia embrionaria, ultrasonografía, hormona FSH.

\begin{abstract}
Yostar, E.J.; Mujíca, I.F.; Stahringer, R.C.; Capellari, A.: Comparison of two protocols for superovulation in Braford embryo donors. Rev. Vet. 32: 2, 146-150, 2021. Embryo transfer is the process of collecting embryos from a given female (donor) and transferring them to other (receptors) to continue gestation. The superovulatory treatments are characterized by induce very variable responses, so it is impossible to estimate the number of embryos recovered by donor. Because the problems related to the great variability of super ovulatory treatments we performed here a preliminary assay including super stimulation on day 3 after synchronizing the new follicular wave. The objective of this study was to evaluate the super ovulatory response to two super stimulation protocols in donors from the Argentine north-east region (NEA). We worked with 60 Braford donors from 5 cow breeders, randomly assigned to two treatments ( $\mathrm{Tl}: \mathrm{n}=30$ and $\mathrm{T} 2$ : $\mathrm{n}=30$ ), in each collections program, 12 donors were utilized. In T1, super ovulation started on day 3 (considering day 0 at the time of placement of progesterone device and $17 \beta$ estradiol) thus shortening the entire treatment and concluding with collection of the embryos on day 14 and not 15 as in T2. The analyzed variables were: total structures subdivided into: transferable embryos, degenerate embryos and unfertilized oocy tes. The number of transferable embryos achieved was $7 \pm 1.19$ and $6 \pm 0.89$, degenerate embryos of $1 \pm 0.32$ and $1 \pm 0.24$ and unfertilized oocytes of $3 \pm 0.86$ and $7 \pm 1.83$ in $\mathrm{T} 1$ and T2, respectively. The independence test showed statistically significant evidence to declare the association between treatments and structures recovered in the collection with a frequency of $62 \%$ for transferable embryos in $\mathrm{T} 1$ and $44 \%$ in $\mathrm{T} 2(\mathrm{p}<0.0001)$. We conclude
\end{abstract}


that although the anticipated use of FSH in ST did not allow to improve super ovulatory response it can be used strategically without affecting the production of embryos.

Key words: bovines, embryo transfer, ultrasonography, hormone FSH.

\section{INTRODUCCIÓN}

Se describe a la transferencia embrionaria (TE) como el proceso de colectar uno o más embriones (EMB), en el inicio de su desarrollo, de una determinada hembra donante (DN) y transferirlos al útero de receptoras fisiológicamente sincronizadas, para dar continuidad a la preñez ${ }^{9}$. El principal uso en bovinos está relacionado con un mejor aprovechamiento de la vía materna en programas de mejoramiento genético.

La selección genómica está siendo utilizada cada vez más para seleccionar las DN ${ }^{29} \mathrm{y}$ testear genéticamente a toros superiores ${ }^{17}$; siendo probados por la producción de sus hermanos, en lugar de sus progenies ${ }^{30}$ . De esta manera los intervalos generacionales se han acortado a 3,5 años en comparación de los 5,5 de las pruebas de progenie tradicionales ${ }^{20}$.

El éxito de la TE se mide por el número de terneros nacidos vivos por $\mathrm{DN}$ en un determinado lapso de tiempo, siendo influenciado por factores como el número de ovulaciones, la fertilización y la viabilidad de los EMB 1. Sin embargo, el mayor problema es la variabilidad en la respuesta a los tratamientos superovulatorios (TS) reflejándose en que un $25-30 \%$ de vacas no producen EMB $^{1,13,18}$.

Existe poca información acerca de los factores genéticos y/o ambientales que afectan la respuesta a los tratamientos superovulatorios (TS) y producción de EMB en razas sintéticas ${ }^{9}$. Los TS disponibles provocan respuestas extremadamente variables ${ }^{12}$ lo que hace imposible estimar el número de EMB recuperados por DN, por lo que se considera un promedio de 5 EMB transferibles, con extremos de 0 a más de $50{ }^{14}$.

Otro de los factores negativos son las altas tasas de ovocitos sin fertilizar, atribuidos a alteraciones de la maduración como consecuencia del desorden bioquímico, metabólico y motriz del oviducto y del útero, afectando el éxito y costo de la técnica ${ }^{26}$. En los últimos 15 años, el promedio de EMB transferibles por colecta ha aumentado de 5,5 a 6,7 a nivel mundial, mejorando un $22 \%^{19}$.

Los avances, como iniciar los TS en momentos al $\operatorname{azar}^{4,6,19}$, el uso de IATF ${ }^{8,21}$, y la reducción del número de aplicaciones de FSH ${ }^{5,10,36}$ han hecho que los protocolos sean más fácil de implementar. Sería interesante saber si esta evolución será capaz, en el futuro, de alterar la variabilidad en las respuestas.

En base a lo expuesto, se propuso comenzar la superovulación (SPO) el día 3 en vez del cuarto día después de la aplicación de $17 \beta$-estradiol y progesterona, como en los protocolos convencionales.

El objetivo fue evaluar la respuesta a dos protocolos, mediante la evaluación de las estructuras recuperadas a la colecta, utilizando el método no quirúrgico a circuito cerrado, en DN Braford del Noreste Argentino (NEA).

\section{MATERIAL Y MÉTODOS}

El experimento se llevó a cabo en cinco cabañas del NEA adheridas al Centro Biotecnológico de Reproducción Bovina (MUNAR y Asociados S.A.). Las DN $(n=60)$ fueron asignadas aleatoriamente (DBCA) a dos tratamientos (T1: $n=30$ y T2: $n=30)$; en cada programa de TE se trabajó con 12 DN (6 para T1 y 6 para T2), considerando a cada uno como unidad experimental y a cada cabaña como un bloque.

Los TS se iniciaron con el control de las ondas foliculares y del folículo dominante comenzando ambos el día 0 con dispositivos intravaginales de progesterona CIDR ${ }^{\circledR}$ (1,9 g de progesterona, Lab. Zoetis, Argentina) $+100 \mathrm{mg}$ de Progesterona ${ }^{\circledR}$ (Lab. Río de Janeiro, Argentina) $+5 \mathrm{mg}$ de $17 \beta$ estradiol (Lab. Río de Janeiro, Argentina) (P4+17ß-e+DIB).

En T1 el día 3 se comenzó la SPO con FSH Folltropin-V® (400 mg NIH-FSH-Pl; Vetrepharm, Canadá Inc.), 8 aplicaciones totales IM a dosis decrecientes, 2 diarias (320 mg totales). El día 5 además de FSH, se aplicó $150 \mu \mathrm{g}(2 \mathrm{ml})$ de prostaglandina sintética, Acceleration-D $\mathbb{B}($ Lab. Vabriela, Argentina).

El día 6 se retiró el dispositivo intravaginal (08:00 h) y el día 7 se detectó celo por la mañana $(08: 00$ h) y por la tarde $(18: 00 \mathrm{~h})$ se aplicó $50 \mathrm{mg}(2 \mathrm{ml})$ de Gonasyn ${ }^{\mathbb{B}}$ (Lab. Syntex, Argentina) y se realizó inseminación artificial con dos dosis (pajuelas) de semen, repitiéndose el día $8(08: 00$ h) con una sola dosis. El día 14 se llevó a cabo la colecta de EMB. A continuación se esquematizan los protocolos correspondientes.

\section{Tratamiento 1:}

\begin{tabular}{|c|c|c|c|c|c|c|c|}
\hline \begin{tabular}{c} 
P4+17ße+DIB \\
\hline 08:00 hs
\end{tabular} & FSH 08:00 hs & FSH 08:00 hs & FSH 08:00 hs & FSH 08:00 hs & 08:00 hs & 08:00 hs & Colecta 08:00 hs \\
\hline Dia 0 & Dia 3 & Dia 4 & Dia 5 & Día 6 & Día 7 & Día 8 & Día 14 \\
\hline & FSH 18:00 hs & FSH 18:00 hs & FSH 18:00 hs & FSH 18:00 hs & GnRH 18:00 hs & \\
\hline & & PGF 18:00 hs & & IATF x 2 dosis & \\
\hline
\end{tabular}


En T2, la SPO comenzó el día 4 y de esta forma toda la secuencia del tratamiento se atrasó 24 h respecto a T1.

\section{Tratamiento 2:}

\begin{tabular}{|c|c|c|c|c|c|c|c|}
\hline P4+17ße+DIB & \multicolumn{3}{c|}{ PGF 08:00 hs } & Retirar DIB & Control Celo & IATF x 2 dosis \\
\hline 08:00 hs & FSH 08:00 hs & FSH 08:00 hs & FSH 08:00 hs & FSH 08:00 hs & 08:00 hs & 08:00 hs & Colecta 08:00 hs \\
\hline Dia 0 & Dia 4 & Dia 5 & Dia 6 & Dia 7 & Día 8 & Dia 9 & Dia 15 \\
\hline & FSH 18:00 hs & FSH 18:00 hs & FSH 18:00 hs & FSH 18:00 hs & GnRH 18:00 hs & \\
\hline & & PGF 18:00 hs & IATF x 2 dosis & \\
\hline
\end{tabular}

Las DN se manejaron en piquetes contiguos al sitio donde se aplicaron los TS, con sombra, reparo, así como agua fresca y limpia. Se controló la alimentación en base a pasturas y/o heno de buena calidad y alimento balanceado. La condición corporal de las mismas al inicio del tratamiento no difirió entre grupos $\bar{X} \pm$ E.E. $=$ $6,85 \pm 0,11$, en escala $1-9)^{15}$

Se administraron suplementos vitamínicos y minerales por vía SC: $5 \mathrm{ml}$ de Cuprhormone ${ }^{\circledR}$ y $10 \mathrm{ml}$ de Selfos-ADE® (Laboratorio Agro Insumos) 10 días antes del inicio de los TS. Se utilizó semen producido en centros de inseminación certificados por SENASA y CABIA, siendo nuevamente analizado previo a su utilización.

Los EMB fueron obtenidos mediante método no quirúrgico a circuito cerrado de flujo discontinuo. Se emplearon filtros (EM-CON囚) estériles con malla de acero inoxidable de $75 \mu \mathrm{m}$ de poro para el filtrado del

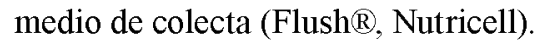

El volumen de la solución conteniendo los EMB $(50 \mathrm{ml})$ se colocó en placas de Petri cuadriculadas, una vez encontrados fueron transportados a una placa más chica con medio Holding ${ }^{\circledR}$ (Nutricell) a fin de lavarlos antes de la transferencia en fresco o la congelación. Una vez que los EMB fueron lavados se realizó la evaluación morfológica de los mismos mediante el uso de lupa estereoscópica $(40 \mathrm{x})$.

Los EMB obtenidos fueron clasificados teniendo en cuenta el estadio de desarrollo y grados de calidad según normas de la International Embryo Transfer Society (IETS) ${ }^{31}$. Para el diseño experimental estadístico se utilizaron bloques completos al azar (DBCA). Los bloques fueron los 5 establecimientos con 12 DN o unidades experimentales. Se realizó estadística descriptiva (media y error estándar).

Las variables analizadas fueron estructuras totales subdivididas en: EMB transferibles, degenerados y ovocitos sin fertilizar. Debido a que las variables estudiadas toman valores alternativos presentando una distribución binomial, se utilizaron tablas de contingencia para el cálculo de ford del NEA. las frecuencias absolutas y relativas y prueba de $\mathrm{Chi}^{2}$ $(\mathbf{p}<0,05)$. Para todos los análisis estadísticos se utilizó software estadístico Infostat ${ }^{11}$

\section{RESULTADOS Y DISCUSIÓN}

Los resultados de la estadística descriptiva para las variables respuesta se presentan en la Tabla 1.

Los EMB transferibles fueron inferiores a los informados por otros autores, de 7,8 $\pm 0,3$ en razas sintéticas Braford y Brangus y 7,5 $\pm 0,6$ en Bonsmara ${ }^{25}$, al igual que en Bonsmara de 7,6 $\pm 0,7^{33}$ y Brangus de 7,7 $\pm 1,1^{34}$; así mismo se mostraron similares a $6,8 \pm 0,6$ en Simmental ${ }^{25}$ y $7,0 \pm 1,7$ en Braford ${ }^{35}$ y superiores a $\mathbf{5 , 7} \pm 0,3{ }^{25}$ y $5,7 \pm 0,6$ en Aberdeen Angus ${ }^{32}$ utilizando $260 \mathrm{mg}$ de Folltropin-V en dosis decrecientes IM cada $12 \mathrm{~h}$, durante 4 días.

Los EMB degenerados obtenidos resultaron inferiores a 3,5 utilizando FSH-P, $3 \mathrm{mg}$ IM dos veces al día por 5 días ${ }^{3}$. Respecto a los ovocitos sin fertilizar se mostraron superiores a 3,19 $\pm 3,09$ en Brahman con un protocolo P-24 similar a T2 ${ }^{28}$. Los EMB congelables fueron inferiores de $6,71 \pm 0,44$; mientras que los transferibles en fresco similares a $0,93 \pm 0,09$ en vacas Nelore tratadas con 8 aplicaciones de FSH ${ }^{23}$

En la Tabla 2 se informa la estadística descriptiva de las variables evaluadas según tratamientos.

Los EMB transferibles fueron similares a los informados de 7,67 $\pm 0,53$, los degenerados superiores de $0,67 \pm 0,17$; los ovocitos sin fertilizar superiores de 0,33

Tabla 1. Media aritmética (x) y error estándar (EE) para EMB transferibles (congelables: E cong. y transferibles en fresco: E. transf. fresco, degenerados (E. deg.), ovocitos sin fertilizar (UFO) y estructuras totales (ET) en DN Bra-

\begin{tabular}{lccccc}
\hline estadístico & \multicolumn{2}{c}{ EMB transferibles } & E deg. & UFO & ET \\
\hline \multirow{2}{*}{$\overline{\mathrm{X}} \pm \mathrm{EE}$} & \multicolumn{2}{c}{$6,85 \pm 0,71$} & $1,00 \pm 0,10$ & $5,32 \pm 1,01$ & $12,53 \pm 1,06$ \\
& E cong. & E transf. fresco & & & \\
& $5,55 \pm 0,71$ & $0,93 \pm 0,15$ & & & \\
\hline
\end{tabular}

Tabla 2. Media aritmética y error estándar (EE) para EMB transferibles (Emb. Transf.), degenerados (Emb. Deg.), ovocitos sin fertilizar (UFO) y estructuras totales (ET) según T1 y T2 en DN Braford del NEA.

\begin{tabular}{lccccc}
\hline tratamiento & estadístico & emb. transf. & emb. deg. & UFO & ET \\
\hline T1 & \multirow{2}{*}{ Media \pm EE } & $7 \pm 1,19$ & $1 \pm 0,32$ & $3 \pm 0,86$ & $12 \pm 1,18$ \\
T2 & $6 \pm 0,98$ & $1 \pm 0,24$ & $7 \pm 1,83$ & $13 \pm 1,77$ \\
\hline
\end{tabular}


Tabla 3. Media aritmética y error estándar (EE) para EMB congelables (E. cong.) y transferibles en fresco (E. trans. fresco) según T1 y T2 en DN Braford del NEA.

\begin{tabular}{lccc}
\hline tratamiento & estadístico & E. cong. & E. transf. fresco \\
\hline T1 & \multirow{2}{*}{ Media \pm E.E. } & $6,33 \pm 1,16$ & $1,10 \pm 0,21$ \\
T2 & & $4,62 \pm 0,84$ & $0,77 \pm 0,22$ \\
\hline
\end{tabular}

Tabla 4: Frecuencias absolutas y relativas de EMB transferibles (E. Transf.), degenerados (E. Deg.), ovocitos sin fertilizar (UFO) y totales. Prueba $\mathrm{Chi}^{2}$ y p-valor según T1 y T2 en DN Braford del NEA.

\begin{tabular}{lccccc}
\hline categoría & T1 & T2 & total & $\chi^{2}$ & p-valor \\
\hline E. Deg. & $31(0,09)$ & $29(0,07)$ & $60(0,08)$ & & \\
E. Transf. & $218(0,62)$ & $192(0,44)$ & $410(0,52)$ & 31,96 & $<0,0001$ \\
UFO & $104(0,29)$ & $215(0,49)$ & $319(0,40)$ & & \\
total & $353(1,0)$ & $436(1,0)$ & $789(1,0)$ & & \\
\hline
\end{tabular}

Tabla 5 (a y b): Frecuencias absolutas y relativas de estadios embrionarios: mórula en precompactación (m.prec.), mórula, blastocito, blastocito mediano (b. med.) y expandido (b. exp.) de calidad excelente, buena y regular; prueba $\mathrm{chi}^{2}$ y p-valor, según T1 y T2 en DN Braford del NEA.

Tabla 5a. Calidad embrionaria (T1).

\begin{tabular}{lccc}
\hline estadio embrionario & excelente & buena & regular \\
\hline M. Prec. & 11,0 & -- & -- \\
Mórula & 790,77 & 190,18 & 50,05 \\
Blastocisto & 990,93 & 70.07 & 00,01 \\
B. Med. & 71,0 & -- & -- \\
B. Exp. & -- & -- & -- \\
\hline
\end{tabular}

Tabla 5b. Calidad embrionaria (T2).

\begin{tabular}{lccccc}
\hline estado embr. & excelente & buena & regular & $\mathrm{X}^{2}$ & p-valor \\
\hline Mórula & 850,83 & 160,16 & 10,01 & 3,14 & 0,2082 \\
Blastocisto & 590,95 & 30,16 & 00,00 & 0,80 & 0,6699 \\
B. Med. & 31,0 & -- & - & 1,60 & 0,2059 \\
B. Exp. & 31,0 & -- & - & - & - \\
\hline
\end{tabular}

$\pm 0,10$ y las estructuras totales también superiores de $8,7 \pm 0,6$ utilizando $\mathrm{FSH}$ a dosis decrecientes, durante 4 días a partir del cuarto día ${ }^{23}$.

La estadística descriptiva para EMB congelables y transferibles en fresco se presenta en la Tabla 3.

Los EMB congelables fueron superiores a los obtenidos por otros autores, de 3,71 $\pm 3,85$ en Brahman con un protocolo P-24 similar a T2 ${ }^{28}$. Los EMB transferibles en fresco resultaron inferiores a los obtenidos de $5,7^{2} ; 5,6^{22}$ y $4,4 \pm 3,87^{28}$, utilizando FSH, eCG y antieCG (200 mg FSH, 2000 UI eCG y 2500 UI anti-eCG).

En la Tabla 4 se presentan las frecuencias absolutas y relativas de la prueba de independencia, según tratamiento, para las estructuras recuperadas en la colecta.

Resultados similares a los obtenidos en Tl de EMB transferibles fueron presentados de $64 \%$ utilizando Folltropin-V (bajo contenido de $\mathrm{LH}$ ) ${ }^{27}$. Sin embargo, se han informado resultados similares a los logrados en $\mathrm{T} 2$, de $37 \%$ trabajando con DN de razas carniceras con protocolos similares ${ }^{7}, \mathrm{y}$ resultados de $18 \%$ cuando utilizaron Folltropin-P (alto contenido de $\mathrm{LH}$ ) ${ }^{27}$.

Los EMB degenerados en ambos casos fueron inferiores a lo informado, de 28 y $13 \%$; mientras que en ovocitos sin fertilizar obtuvieron $8 \%$ y $68 \%$, utilizando Folltropin$\mathrm{V}$ (bajo contenido de LH) y con Folltropin-P (alto contenido de $\mathrm{LH}$ ) superiores a lo logrado en este ensayo ${ }^{27}$

Por último se presentan los resultados de la prueba de independencia para calidad y estadios embrionarios (Tabla 5)

El estadio mórula fue superior de 20 y $31 \%$, los blastocitos similares a 40 y $54 \%$ en Holstein usando (Folltropin $\mathbb{R}$ ) con y sin tratamiento de somatotrofina bovina (bST), respectivamente ${ }^{24}$. Otros trabajos lograron obtener una mayor cantidad de EMB en los estadios de mórula, blastocito temprano y blastocito con un $37 ; 30$ y $23 \%$, respectivamente, y en menor grado blastocitos expandidos con un $6 \%{ }^{16}$.

Así también se reporta predominancia de los estadios mórula, blastocito temprano y blastocito con un 32,$9 ; 26,4$ y $19,1 \%$ respectivamente, y en menor grado $0,9 \%$ de blastocitos expandidos y $0,4 \%$ de blastocitos eclosionados ${ }^{26}$, siguiendo los mismos patrones porcentuales que el presente trabajo

Los EMB de calidad excelente se presentaron con una frecuencia similar a 85 y $84 \%$, los de buena calidad fueron inferiores a $15 \%$ y los de calidad regular también inferiores de $16 \%$ en DN superovuladas (con Folltropin $($ )) similar a T2, con y sin tratamiento con somatotrofina bovina (bST) ${ }^{24}$.

Concluimos que bajo las condiciones en las que se realizó el ensayo, el uso anticipado de FSH en los protocolos de SPO en DN Braford del NEA no permitió mejorar la respuesta superovulatoria evaluada mediante el total de estructuras y/o la calidad de los EMB recuperados en la colecta, respecto a los tratamientos convencionales. Sin embargo, puede ser utilizado cuando por razones estratégicas (agenda, infraestructura, caminos, clima, disponibilidad de personal) sea necesario, sin afectar la producción de EMB, acortando los tratamientos convencionales.

\section{REFERENCIAS}

1. Armstrong DT. 1993. Recent advances in super ovulation of cattle. Theriogenology 39: 7-24.

2. Baruselli PS et al. 2003. Adequação da dose de FSH (Folltropin-V) em protocolos de superovulação de vacas nelore (Bos taurus indicus) com inseminação artificial em tempo fixo (SOTF). Acta Sci Vet 31: 244-245. 
3. Becker WA, Pinheiro LE. 1986. Ovarian response to super ovulation in Nelore cows (Bos Taurus indicus). Theriogenology. 25: 785-793.

4. Bergfelt DR, Bó GA, Mapletoft RJ, Adams GP. 1997. Super ovulatory response following ablation-induced follicular wave emergence at random stages of the oestrus cycle in cattle. Anim Reprod Sci 49: 1-12.

5. Bó GA, Hockley DK, Nasser LF, Mapletoft RJ. 1994. Superovulatory response to a single subcutaneous injection of a porcine pituitary extract in beef cattle. Theriogenology 42: 963-975.

6. Bó GA et al. 1995. Ovarian follicular wave emergence after treatment with progestogen and estradiol in cattle. Anim Reprod Sci 39: 193-204

7. Bó GA, Adams GP, Pierson RA, Mapletoft RJ. 1996. Effect of progestogen plus estradiol-17P treatment on superovulatory response in beef cattle. Theriogenology. 45: 897-910.

8. Bó GA, Baruselli PS, Chesta P, Martins CM. 2006. The timing of ovulation and insemination schedules in super stimulated cattle. Theriogenology 65: 89-101.

9. Cardoso JC. 2002. Fatores que influenciam a produção de embriões de vacas nelore (B. taurus indicus) superovuladas.UNESP, São Paulo, Brasil, 46 p.

10. Carvalho PD et al. 2014. Use of a single injection of long acting recombinant bovine FSH to super ovulate Holstein heifers: a preliminary study. Theriogenology 82: 3, 481489.

11. Dirienzo JA et al. 2014. InfoStat versión 2014. Grupo InfoStat, FCA, Universidad Nacional de Córdoba, Argentina. URL http://www.infostat.com.ar

12. Donaldson LE. 1984. The day of the estrus cycle that FSH is started and superovulation in cattle. Theriogenology 22 97-99.

13. Hahn J. 1992. Attempts to explain and reduce variability of super ovulation. Theriogenology 38: 269-275.

14. Hasler JF. 1992. Current status and potential of embryo transfer and reproductive technology in dairy cattle. $J$ Dairy Sci 75: 2857-2879.

15. Herd DB, Sprott LR. 1986. Body condition, nutrition and reproduction in beef cows. Bullet Agric Ext Serv, Texas A\&M University, 1526: $11 \mathrm{p}$.

16. Lindner GM, Wright RW. 1983. Bovine embryo morphology and evaluation. Theriogenology 20: 4, 407-416

17. Lohuis MM. 1995. Potential benefits of bovine embryomanipulation technologies to genetic improvement programs. Theriogenology 43: 51-60.

18. Looney CR, Oden AJ, Massey JM, Johnson CA, Godke RA. 1984. Pregnancy rates following hCG administration at the time of transfer in embryo-recipient cattle. Theriogenology 21: 246-247.

19. Mapletoft RJ, Bó GA. 2012. The evolution of improved and simplified superovulation protocols in cattle. Reprod Fert Dev 24: 1, 278-283.

20. Mapletoft RJ. 2015. Estado actual y perspectivas de las biotecnologías reproductivas en los próximos 10 años. $X I$ SIRA (Córdoba, Argentina), p. 31-42.

21. Martinez MF, Kastelic JP, Adams GP, Mapletoft R. 2002. The use of a progesterone releasing device (CIDR-
B) or melengestrol acetate with GnRH, LH or estradiol benzoate for fixed time AI in beef heifers. J Anim Sci 80: 1746-1751.

22. Martins CM, Marques MO, Silva RC, Baruselli PS. 2005. Adequação do protocolo de superovulação com inseminação artificial em tempo fixo em Bos taurus. Tesis de Maestría, Universidade de Sao Paulo, Brasil.

23. Martins CM et al. 2008. Efeito da redução do número de administrações de FSH na resposta super ovulatória e na produção de embriões de doadoras nelore. Acta Sci Vet in press.

24. Moreira F, Badinga L, Burnley C, Thatcher WW. 2002 Bovine somatotropin increases embryonic development in super ovulated cows and improves post transfer pregnancy rates when given to lactating recipient cows. Theriogenology 57: 4, 1371-1387.

25. Ochoa JC, Ramirez RR, Piccardi MB, Bó GA, Tríbulo H. 2009. Influencia de la estación en la producción de embriones en donantes de embriones de raza para carne. VIII SIRA, Córdoba, Argentina.

26. Palma G, Brem G. 2001. Biotecnología de la Reproducción, Cap. I, Edit. INTA Balcarce, Argentina, p.1-19.

27. Quaresma MA, Lopes CL, Robalo SJ. 2003. Superovulation of Mortelenga cows with two FSH preparations (FSH-P \& Folltropin). Rev Portug Cienc Vet 98: 81-84.

28. Salgado OR, Mejía AA, Suarez PS. 2011. Eficiencia de la respuesta superovulatoria del ganado Brahman al protocolo p-24. Rev MVZ Córdoba 16: 2, 2521-2527.

29. Seidel GE. 2010. Brief introduction to whole genome selection in cattle using single nucleotide poly-morphisms ReprodFert Dev 22: 138-144.

30. Smith C, Ruane J. 1987. Use of sib testing as a supplement to progeny testing to improve the genetic merit of commercial semen in dairy cattle. Can J Anim Sci 67: 985990.

31. Stringfellow DA, Seidel SM. 1998. Manual of the International Embrio Transfer Society: a procedural guide and general information for the use of embryo transfer technology, emphasizing sanitary precautions, $3^{\text {rd }}$ ed., Ed. Savoy, Illinois (USA), $170 \mathrm{p}$.

32. Tríbulo A et al. 2009. Efecto de la dosis y protocolo de administración de Folltropin- $V$ en la producción de embriones de donantes Angus. VIII SIRA, Córdoba, Argentina.

33. Tríbulo A et al. 2009. Efecto de la dosis y protocolo de administración de Folltropin- $V$ en la producción de embriones de donantes Bonsmara. VIII SIRA, Córdoba, Argentina.

34. Tríbulo A et al. 2009. Efecto de la dosis y protocolo de administración de Folltropin- $V$ en la producción de embriones de donantes Brangus. VIII SIRA, Córdoba, Argentina.

35. Tríbulo A et al. 2009. Superovulación de donantes Braford con una dosis de Folltropin-V. VIII SIRA, Cordoba, Argentina.

36. Tríbulo A, Rogan D, Tríbulo H, Mapletoft R, Bó GA. 2012. Superovulation of beef cattle with a split single intramuscular administration of Folltropin- $V$ in two concentrations of hyaluronan. Theriogenology 77: 8, 1679-1685. 